\title{
Phlebologie
}

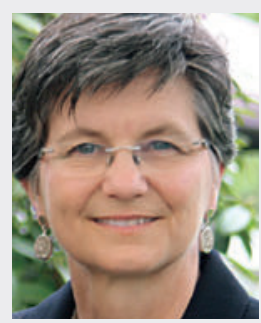

Dr. med. Erika Mendoza

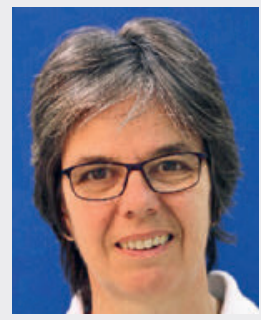

Prof. Dr. med. Birgit Kahle

\section{Schmerz in der Phlebologie?}

Liebe Leserinnen und Leser,

Beinschmerz ist eine der Haupt-Ursachen, warum Patienten unsere Sprechstunden oder Krankenhäuser aufsuchen. Wenngleich ihre möglicherweise vorhandenen Krampfadern bereits seit Jahren sichtbar sind, fallen sie dem Patienten oder Zuweiser oft erst in Zusammenhang mit dem Schmerzereignis auf. Daher ist die Phlebologie häufig die erste Anlaufstelle bei Beinschmerzen. In Abhängigkeit von der (auch dieser unkritischen Inanspruchnahme geschuldeten) Überlastung der Sprechstunden, warten die Patienten dann Monate, bis sie gesagt bekommen, dass ihr Schmerz nicht venös bedingt sein kann.

Durch Medien verstärkte Fokussierung auf Beinschmerzen im Rahmen einer Adipositas führen auch vermehrt Patientinnen mit der Frage nach einem Lipödem in die Praxen. Dieser Aspekt ist aktuell sehr relevant, so verwandelt der Schmerz möglicherweise die Adipositas oder die Lipohypertrophie in ein Lipödem. Der real vorhandene Schmerz - oder aber auch der suggerierte, in der Hoffnung, dass dann das unschöne Beinbild mit Liposuktion behandelt werden kann - der unsere Sprechstunden mit Forderungen nach Bescheinigungen beansprucht, kommt in diesem Heft nicht zu Wort, wurde aber umfangreich besprochen in vorangehenden Ausgaben.

Auf der anderen Seite sind es auch die Schmerzen, die auf eine Thrombose hinweisen können und daher sehr kurzfristig untersucht und behandelt werden müssen, besonders, wenn sie akut, einseitig und mit Beinschwellung und Verfärbung einhergehen bei einer tiefen Thrombose oder mit Verhärtungen, Verfärbung und Druckschmerz entlang vorhandener oberflächlicher Krampfadern bei der oberflächlichen Thrombose. In diesem Punkt ist Aufklärung der Bevölkerung und Fokussierung seitens der durch die Ängste der Patienten verunsicherten Zuweiser erforderlich.

Der Beinschmerz ist in diesem Heft Thema und wird aus verschiedenen Aspekten beleuchtet - dermatologische, venöse, arterielle und orthopädische Ursachen, sowie eine Orientierung zur Schmerztherapie.

Wir wünschen dem Leser hilfreiche Hinweise aus dieser Zusammenstellung!

Dr. med. Erika Mendoza

Prof. Dr. med. Birgit Kahle 